\title{
ON THE $p$-ELEMENTS OF A FINITE GROUP
}

\author{
C. Y. HO
}

ABSTRACT. Let $G$ be a finite group, $p$ a prime, and $x$ a $p$-element in $G$. An element $g$ in $G$ is called a witness of $G$ if the subgroup generated by $x$ and $g$ is a $p$-group. The set of all witnesses of $x$ in $G$ is denoted by $W(x)$. This paper shows that $x$ belongs to a given Sylow $p$-subgroup $P$ of $G$ if one of the following holds: (1) $G$ is $p$-solvable and $W(x) \supset P \cap\left\{\left.x^{g}\right|_{g} \in G\right\}$; (2) $G$ is $p$-solvable, $P=\langle P \backslash Z(P)\rangle$, and $W(x) \supset P \backslash Z(P)$; (3) $\operatorname{cl}(P) \leq 2$ and $W(x) \supset P$; (4) $x$ normalizes a subgroup $P_{1}$ of $P$ with $\left|P: P_{1}\right| \leq p^{2}$ and $W(x) \supset P ;(5)|P|=p^{4}$ and $W(x) \supset P$.

1. Introduction and notation. Let $G$ be a finite group, $p$ a prime number, and $x$ a $p$-element in $G$. An element $g$ in $G$ is called a witness of $x$ if the subgroup generated by $x$ and $g$ is a p-group. The set of all witnesses of $x$ in $G$ is denoted by $W(x)$.

It is interesting to know how $W(x)$ reflects the property of $x$. For example, it is interesting to see which property of $W(x)$ will imply that $x$ belongs to the maximal normal p-subgroup of $G$, and which property of $W(x) \cap H$ will imply that $x$ belongs to $H$, where $H$ is a given $p$-subgroup of $G$.

When $W(x)$ contains the conjugacy class of $x$, Baer's the orem tells us that $x$ belongs to the maximal normal $p$-subgroup of $G$.

This paper will show that $x$ belongs to a given Sylow $p$-subgroup when certain conditions are imposed on the Sylow $p$-subgroup and $W(x)$. This is summarized in

Theorem 1. Let $G$ be a finite group and $P$ a Sylow p-subgroup of $G$. For $x \in G$, if one of the following bolds, then $x \in P$.

(1) $G$ is p-solvable and $W(x) \supset P \cap\left\{x^{g} \mid g \in G\right\}$.

(2) $G$ is p-solvable, $P=\langle P \backslash Z(P)\rangle$, and $W(x) \supset P \backslash Z(P)$.

Received by the editors November 12, 1973 and, in revised form, January 28, 1974. AMS (MOS) subject classifications (1970). Primary 20F 45, 20F 15; Secondary $20 \mathrm{~F} 03$.

Key words and phrases. Largest solvable normal subgroup, center, nilpotent, nilpotent class, Fitting subgroup, Frattini subgroup, $p$-solvable, simple group, socle, Sylow $p$-subgroup, witness. 
(3) $\mathrm{cl}(P) \leq 2$ and $W(x) \supset P$.

(4) $x$ normalizes a subgroup $P_{1}$ of $P$ with $\left|P: P_{1}\right| \leq p^{2}$ and $W(x) \supset P$.

(5) $|P|=p^{4}$ and $W(x) \supset P$.

Some examples are given in the final section which show that the conclusion of Theorem 1 becomes invalid when the stated conditions are relaxed.

All groups in this paper are assumed to be finite. Our notation is standard and taken mainly from [1]. In particular, let $G$ be a group. Then Soc $(G)$, Sol $(G), O_{p}(G), F(G), \Phi(G), \exp (G)$ and $G^{\prime}$ denote respectively the joint of all minimal normal subgroups, the largest solvable normal subgroup, the maximal normal p-subgroup, the Fitting subgroup, the Frattini subgroup, the exponent and the commutator subgroup of $G$. Moreover, for any nilpotent group $G, \mathrm{cl}(G)$ denotes the nilpotent class of $G$.

2. Proof of Theorem 1. Suppose $X$ is an arbitrary finite group. If $\operatorname{Sol}(x)=1$, then it is well known that $C_{X}(\operatorname{Soc}(X))=1$. This can be seen from the following. Since $\operatorname{Sol}(X)=1$, Soc $(X)=S_{1} \times \cdots \times S_{n}$, where each $S_{i}$ is a nonabelian group. Thus $C_{X}(\operatorname{Soc}(X)) \cap \operatorname{Soc}(X)=1$. However, $C_{X}(\operatorname{Soc}(X)) \triangleleft X$ which, together with the fact that $\operatorname{Sol}(X)=1$, implies $C_{X}(\operatorname{Soc}(X))=1$.

We use induction on $|G|$ to prove Theorem 1; assume that Theorem 1 is false. Hence $O_{p}(G)=1$ and $G=\langle P, x\rangle$. Let $K$ be the conjugacy class containing $x$ and let $S$ be any minimal normal subgroup of $G$. By induction we can see that $G=P S=S P$. Let $B(x)=\{X \mid X=\langle x, A\rangle$ is a $p$-group and $A \supset P\}$. Choose $R \in B(x)$ such that $|R \cap P|$ is of maximal order.

Cases (1) and (2). Since $G$ is $p$-solvable and $O_{p}(G)=1, S$ is a normal subgroup of order relative prime to $p$. Let $x^{s} \in K \cap P$ for some $s \in S$. If $P \cap K \subset W(x)$, then $\left\langle x^{s}, x\right\rangle$ is a $p$-group. However $x^{-1} x^{s}=$ $[x, s] \in S$. Therefore $W(x) \supset P \cap K$ implies $[x, s]=1$, and so $x=x^{s} \in P$. This proves (1).

Now suppose Case (2) occurs. For $g \in G$, we have $g=a(g) \cdot b(g)$, where $a(g) \in S$ and $b(g) \in P$. Hence $x^{g} \in P \backslash Z(P)$ if and only if $x^{a(g)} \in P \backslash Z(P)$. Since $[x, a(g)]=x^{-1} x^{a(g)} \in S, K \cap P \subset Z(P)$. Hence $\left[x^{s}, y\right]=1$ for all $y \in P$. As

$$
\left[x^{s}, y\right]=[x[x, s] y]=[x, y]^{[x, s]}[[x, s], y] \text {, }
$$

$[x, y]=\left([[x, s], y]^{-1}\right)^{[x, s]^{-1}}$. Since $S \triangleleft G$ and $W(x) \supset P \backslash Z(P),[x, y]=1$ for all $y \in P \backslash Z(P)$. As $\langle P \backslash Z(P)\rangle=P,[x, P]=1$, which is impossible. This contradiction completes the proof of Case (2). 
Case (3). By (1) we may assume that $\operatorname{Sol}(G)=1$. Hence Soc $(G)=S$. Let $F=\{[y, x] \mid y \in P\}$. If $F=1$, then $x \in P$. Therefore $1 \neq$ $\langle F\rangle \subset C_{G}(x)$. For any $y, z \in P$, we have $[y, x]^{z}=[y z, x][z, x]^{-1}$. This shows $P \subset N_{G}(\langle F\rangle)$. Hence $\langle F\rangle \triangleleft G$. Since Sol $(G)=1, C_{G}(\operatorname{Soc}(G))=1$. Thus $S \subset\langle F\rangle$. Now $x \in C_{G}(S)$ implies $x=1$. Of course $1 \in P$, a contradiction. This completes the proof of (3).

Case (4). The case $\left|P: P_{1}\right|=p$ is trivial. Hence we may assume that $\left|P: P_{1}\right|=p^{2}$. Therefore there exists an element $y \in N_{P}\left(P_{1}\right) \backslash P_{1}$ such that $\langle x, y\rangle$ is a $p$-group. Since $\langle x, y\rangle \subset N_{G}\left(P_{1}\right),\left\langle x, y, P_{1}\right\rangle \in B(x)$. So $|R \cap P| \geqslant\left|P_{1}\right|$. This shows that $R \cap P$ is a maximal subgroup of any Sylow $p$-subgroup containing it. Clearly $x$ normalizes $R \cap P$ and an application of (3) completes the proof of (4).

Case (5). By (3) and (4) we may assume that $\mathrm{cl}(P)>2$ and $|R \cap P| \ngtr p^{2}$. Therefore $P^{\prime} \not \subset Z(P), P^{\prime}=\Phi(P)$, and $P / P^{\prime} \simeq Z_{p} \times Z_{p}$. Suppose $\exp (P)=p^{2}$. Let $y \in P$ be an element of order $p^{2}$ and let $D=\langle y\rangle$. We may assume that $R \cap P=D$. Suppose $x \in N_{G}(D)$. Let $y_{1} \in N_{P}(D) \backslash D$. Then $\left\langle x, D, y_{1}\right\rangle \in B(x)$, which violates the maximality of $R$. Therefore $x \notin N_{G}(D)$, and $R=\langle y, x\rangle$ is a Sylow $p$-subgroup of $G$. Let $N=N_{R}(D)$. Then $N \neq R$ and $N \supsetneqq D$. Since $N^{x}=N$ and $|N|=p^{3}, D \cap D^{x}=E$, where $E$ is the unique subgroup of order $p$ in $D$. So $x$ centralizes $E$. If $D \triangleleft P$, then $E \subset Z(P)$, which implies $E \subset Z(G)$, a contradiction. So $D \& P$. Let $N_{1}=N_{P}(D)$ and let $u \in P \backslash N_{1}$. Then $N_{1}=N_{1}^{u}$ and so $D \cap D^{u}=E$. This implies $E \subset Z(P)$ which is impossible. Hence $\exp (P)=p$. If $p=2$, then $P$ is abelian, a contradiction. Hence $p$ is odd. Let $A$ be a maximal abelian subgroup of $P$ containing $P^{\prime}$. If $A \neq P^{\prime}$, then $A \simeq Z_{p} \times Z_{p} \times Z_{p}$, and $P=A\langle y\rangle$ with $y^{P}=1$. We can view $A$ as a 3-dimensional vector space over the finite field of $p$-elements, and $y$ a linear transformation on $A$. With respect to a suitable basis $\left\{v_{1}, v_{2}, v_{3}\right\}$ of $A, y^{-1}$ has the matrix representation

$$
\left(\begin{array}{lll}
1 & 1 & 0 \\
0 & 1 & 1 \\
0 & 0 & 1
\end{array}\right) .
$$

Hence $v_{1}^{y^{-1}}=v_{1} v_{2}$ and $v_{2}^{y^{-1}}=v_{2} v_{3}$. So

$$
\begin{aligned}
\left(y v_{1}\right)^{p} & =\left(y v_{1} y^{-1}\right)\left(y^{2} v_{1} y^{-2}\right) \cdots\left(y^{(p-1)} v_{1} y^{-(p-1)}\right) y^{p-1} \cdot y v_{1} \\
& =v_{1}^{y^{-1}} \cdot v_{1}^{y^{-2}} \cdots v_{1}^{y^{-(p-1)}} \cdot v_{1} .
\end{aligned}
$$

Let $z=y^{-1}$. Using the linear transformation notation, from $z=1$, we see that $v_{1}^{(z-1)^{p}}=1$. Therefore 


$$
1=v_{1}^{z+z^{2}+\cdots+z^{p-1}+1}=\left(v_{1}^{(z-1)}\right)^{-1}=v_{2}^{-1} \text {. }
$$

This is impossible. Hence $P^{\prime}$ is a maximal abelian subgroup of $P$. So $P^{\prime}=C_{P}\left(P^{\prime}\right)$, and $P / P^{\prime}$ is isomorphic to a subgroup of Aut $\left(P^{\prime}\right)$. Since the Sylow $p$-subgroup of $\mathrm{Gl}(2, P)$ has order $p,|P|=p^{3}$, a contradiction. This completes the proof of (5).

Remark. The proof of (5) also shows that a $p$-group $P$ of order $p^{4}$ with $\exp (P)=p$ is not of maximal class.

A stronger version of (3) in The orem 1 is the following.

Proposition 1. Let $H$ be a nilpotent subgroup of a finite group $G$ and let $x \in G$. If for any $t \in H,\langle x, t\rangle$ is a nilpotent group of nilpotent class not greater than 2 , then $\langle H, x\rangle$ is nilpotent.

Proof. We apply induction to $|G|$. We may assume that $G=\langle H, x\rangle$. Let $S$ be a minimal normal subgroup. By induction, $G / S$ is nilpotent. Set $F=\{[t, x] \mid t \in H\}$. For any $t_{1}, t_{2} \in H$ we have $\left[t_{1}, x\right]^{t} 2=\left[t_{1} t_{2}, x\right]\left[t_{2}, x\right]^{-1}$. Therefore $\langle F\rangle \triangleleft G$ and $x \in C_{G}\langle F\rangle$. If $F=\{1\}$, then the conclusion of the proposition holds. Hence we may assume $F \neq\{1\}$.

Suppose $\operatorname{Sol}(G)=1$. Then $S=\operatorname{Soc}(G)$. Since $C_{G}(\operatorname{Soc}(G))=1$ and $\langle F\rangle \triangleleft G, S \subset\langle F\rangle$. Therefore $x \in C_{G}(S)$, and so $x=1$. Of course $\langle H, x\rangle$ is a nilpotent group in this case.

If $\operatorname{Sol}(G) \neq 1$, then we may assume that $S$ is an elementary abelian $p$-group. Therefore $G$ is solvable. By induction we may assume that $S=F(G)$. By $\left[1\right.$, p. 218], $C_{G}(S)=S$. Therefore $S \subset\langle F\rangle$, and so $x \in S$. Hence $G=H S$. For any prime number $q \neq p, x$ centralizes every $q$-element of $H$. Since $H$ is nilpotent, $H$ is a $p$-group. Hence $G$ is a $p$-group. This completes the proof of Proposition 1.

3. Some examples. If we remove the condition $\langle P \backslash Z(P)\rangle=P$ in (2) of Theorem 1, then any $G$ with more than one nontrivial abelian Sylow p-subgroup will be a counterexample. The following is a less trivial example in this direction.

Example (a). Let $V$ be a 3-dimensional vector space over the field $F$ with four elements. Choose a basis for $V$ which we use to identify the elements in $\mathrm{Gl}(V)$ by its representing matrices. Let

$$
A=\left(\begin{array}{lll}
0 & 1 & 0 \\
0 & 0 & 1 \\
1 & 0 & 0
\end{array}\right), \quad B=\left(\begin{array}{lll}
f & 0 & 0 \\
0 & 1 & 0 \\
0 & 0 & 1
\end{array}\right)
$$


where $f \in F \backslash\{0,1\}$ and $f^{3}=1$. Then $A^{3}=B^{3}=1$ and $\langle A, B$, is a 3-group. Let $G=$ $V\langle A, B\rangle$ be the semidirect product of $V$ and $A, B$ such that for $v \in V$ and $T \in\langle A, B\rangle$, $v^{T}$ is defined to be $v+v(T-1)$. In $G$ we use the multiplicative notation. Let

$$
C=B^{A}=\left(\begin{array}{lll}
1 & 0 & 0 \\
0 & f & 0 \\
0 & 0 & 1
\end{array}\right)
$$

After a short calculation we see that

$$
C_{V}(C) \cap\left[\left(C_{V}(A) \backslash C_{V}(C)\right) \cdot\left(C_{V}(B) \backslash C_{V}(C)\right)\right]
$$

is not empty. Let $v_{1} \in C_{V}(A) \backslash C_{V}(C), v_{2} \in C_{V}(B) \backslash C_{V}(C)$ and $v_{1} v_{2} \in C_{V}(C)$. Hence $1=\left[v_{1} v_{2}, C^{-1}\right]=\left[v_{1}, C^{-1}\right]^{v} 2\left[v_{2}, C^{-1}\right]$. Since $V$ is an elementary abelian 2-group, $\left[v_{1}, C^{-1}\right]=\left[v_{2}, C^{-1}\right]$. Let $v=\left[v_{1}, C^{-1}\right] \neq 1$. Then $\langle v C, C\rangle$ is not a 3-group. But $\langle v C, A\rangle^{v_{1}}=\left\langle v C^{v}, A\right\rangle=\left\langle v\left[v_{1}, C^{-1}\right] C, A\right\rangle=\langle C, A\rangle$ is a 3-group. Similarly $\langle v C, B\rangle^{v} 2=\langle C, B\rangle$ is a 3-group. This shows that although $\langle\nu C, A\rangle,\langle v C, B\rangle$ are 3-groups and $P=\langle A, B\rangle$ is a Sylow 3-subgroup of $G, v C \notin\langle A, B\rangle$. Of course $G$ is 3-solvable. Since $Z(\langle A, B\rangle)$ $=\left\langle B B^{A} B^{A^{2}}\right\rangle, P=\langle P \backslash Z(\langle A, B\rangle)\rangle$. Also $|P|=3^{4}$ in this example. Therefore it can be used to show that if we replace the stated condition in (5) by $W(x)$ which contains some set of generators of $P$, then the conclusion is false.

Example (b). Let $p$ be a prime and let $F$ be the finite field with $p$ elements. Let $G$ be the group of all $3 \times 3$ invertible matrices with determinant 1 over $F$.

\section{Suppose}

$$
x=\left(\begin{array}{lll}
1 & 0 & 0 \\
1 & 1 & 0 \\
0 & 0 & 1
\end{array}\right), \quad y=\left(\begin{array}{lll}
1 & 0 & 1 \\
0 & 1 & 0 \\
0 & 0 & 1
\end{array}\right), \quad \text { and } \quad z=\left(\begin{array}{lll}
1 & 0 & 0 \\
0 & 1 & 0 \\
0 & 1 & 1
\end{array}\right)
$$

Then $G=\langle x, y, z\rangle$ and $P=\langle y, z\rangle$ is a Sylow $p$-subgroup of $G$. Clearly $\mathrm{cl}(P)=2$. It is not difficult to see that $\langle x, y\rangle,\langle x, z\rangle$ are both $p$-groups. Of course $x \notin P$. This shows that if we just require that $\langle x, y\rangle$ is a $p$-group for $y$ belonging to a given set of generators of $P$, then the conclusion of (2) might be false. 
66 C. Y. HO

The author is indebted to Dr. S. Sidki for suggesting Example (a) in this section.

\section{REFERENCE}

1. D. Gorenstein, Finite groups, Harper and Row, New York, 1968. MR 38 $\# 229$.

INSTITUTE OF MATHEMATICS, ACADEMIA SINICA, REPUBLIC OF CHINA

DEPARTAMENTO DE MATEMATICA, UNIVERSIDADE DE BRASILIA, 70,000-BRASILIA, D.F., BRASIL (Current address) 